\title{
Benefit of chemotherapy based on platinum with definitive radiotherapy in older patients with locally advanced esophageal squamous cell carcinoma
}

Haishan $\mathrm{Wu}^{\dagger}$, Yilin Yư ${ }^{\dagger}$ Qunhao Zheng, Tianxiu Liu, Yahua Wu, Zhiping Wang, Hongying Zheng, Lingyun Liu and Jiancheng Li ${ }^{*}$ (D)

\begin{abstract}
Objective: There is still no definitely therapeutic evidence of a beneficial effect of chemotherapy with radiotherapy for older patients with esophageal squamous cell carcinoma (ESCC). We aim to determine the influence of chemoradiotherapy (CRT) and radiotherapy (RT) alone in patients aged 65 years or older with locally advanced ESCC.

Methods: We retrospectively analyzed 581 ESCC patients who underwent CRT and RT alone. Univariate and multivariate Cox regression analysis was used to analyze the impact of clinical factors on long-term overall survival (OS) and progression-free survival (PFS). Finally, we compared the toxicity rates of these patients.

Results: The median OS and PFS of the overall population were 23.2 months (2.0-162.6 months) and 18.6 months (1.1-159.6 months). Multivariate Cox regression analysis showed that chemotherapy $(p<0.05)$, tumor thickness $(p<0.01)$, and $\mathrm{N}$ stage $(p<0.05)$ were independent prognostic factors associated with both OS and PFS. In the chemotherapy subgroup, patients who received 2-8 cycles of chemotherapy had better OS than those who received 1 cycle $(p=0.015)$. The results also revealed that the CRT group has better OS and PFS than RT alone group for patients aged $65-74$ years (both $p<0.01$ ). However, for patients aged 75 years or older, there was no statistically significant difference between CRT and RT alone (both $p>0.05$ ). Besides, higher staged ESCC has the inferior OS and PFS than lower staged ESCC for patients received RT alone and aged 65-74 years (both $p<0.05$ ). Finally, there were significantly more severe hematologic toxicities in the CRT group than in those treated with RT alone in this study $(p<0.001)$.

Conclusions: The present study suggested that CRT for locally advanced ESCC in patients aged 65 years or older had a significant benefit over RT alone in terms of OS and PFS. However, for patients aged 75 years or older, there was no statistically significant difference between CRT and RT alone. CRT should be performed with special attention in patients aged 75 years or older.
\end{abstract}

Keywords: Esophageal squamous cell carcinoma, Chemoradiotherapy, Radiotherapy, Overall survival, Progressionfree survival

*Correspondence: jianchengli_jack@126.com

${ }^{\dagger}$ Haishan Wu and Yilin Yu contributed equally as first authors to this manuscript

Fujian Medical University Cancer Hospital, Fujian Cancer Hospital, Fuzhou 350014, China

\section{Introduction}

Esophageal carcinoma remains one of the most common cancers worldwide and is considered a serious malignant tumor in terms of prognosis and mortality rate. Every year, more than 550,000 new cases of esophageal 
carcinoma (EC) are diagnosed [1, 2]. It occurs mainly in the middle-aged and older individuals with a mean age of 67 years at diagnosis and about $30 \%$ of patients older than 75 years3-5. The number of older populations is increasing rapidly in the world $[6,7]$. Due to the prolongation of life expectancy and aging of the global population increase, older patients with EC are increasing gradually over the recent decades. Cancer is the primary cause of death in older people. Therefore, this is sure to become a major challenge and an increasingly common social issue in the near future.

Despite many advances in surgical techniques, esophagectomy is associated with significant postoperative morbidity and mortality. The increased risk may be more vital in older patients due to a decreased physiological function, higher burden of comorbidities, and more inferior nutritional status $[8,9]$. The older is often considered the limit for this type of surgery. There is a lack of evidence-based evidence regarding the appropriate treatment for the older population with locally advanced esophageal squamous cell carcinoma (ESCC). For patients who are either deemed medically inoperable or have unresectable tumors, the efficacy of definitive chemoradiotherapy (dCRT) has been proved in some randomized, controlled trials for EC patients10-12. However, older patients are under-represented in most randomized trials. Only a few studies have reported the efficacy and safety in elderly patients, thus questioning the feasibility of the results in the older population [13, 14]. Besides, most older patients are unlikely to be able to tolerate chemotherapy. Whether the treatment regime of younger patients can be applied to older patients is still controversial, and little data are available.

As the older population increases, it becomes even more critical to understand whether a standard approach should be used in the treatment of this challenging group of patients. To our knowledge, no high-level evidence specifically addressing the outcome of older patients treated by dCRT approaches has been published so far. Therefore, the standard therapy regimen for the older locally advanced ESCC patients has not reached a consensus yet. The aim of the present study was designed to retrospectively evaluate our hospital's experience among the older patients with locally advanced ESCC who were treated with dCRT to better understand the feasibility, efficacy, and safety of this approach.

\section{Materials and methods}

\section{Study population}

A retrospective study was performed at the Fujian Provincial Cancer Hospital. All consecutive cases of 581 patients aged 65 or older with locally advanced esophageal squamous cell carcinoma (ESCC) who received definitive chemoradiotherapy (dCRT) from December 2007 to August 2020 were included. Patients' inclusion criteria included: (A) aged 65 and older; (B) histopathologic proof of esophageal squamous cell carcinoma (ESCC); (C) Karnofsky Performance Status (KPS) $\geq 70$ points; (D) no distinct metastasis or multiple primary diagnoses; (E) had not undergone esophagectomy; (F) no other major diseases (renal failure, liver failure and severe cardiovascular and cerebrovascular diseases). All the patients were diagnosed with locally advanced ESCC (stage II-IVA). The blood biochemical data was collected within three days before therapy. The tumor length was defined by barium esophagography and/or esophagoscopy, and tumor diameter by computed tomography (CT) scan and/or endoscopic ultrasound. For clinical staging, the eighth tumor-node-metastasis (TNM) edition was performed in all patients for esophageal carcinoma (EC). This study was carried out according to the declaration of Helsinki principles and approved by our Institutional Ethics Board (K2021-086-01).

\section{Radiotherapy}

For treatment planning, the tumor volume was delineated using all available diagnostic information (barium esophagography, esophagoscopy, endoscopic ultrasound, $\mathrm{CT}$, and positron emission tomography (PET)-CT). All plans were completed in the treatment plan system (Philips Pinnacle, USA). CT-based radiation planning and intensity modulated radiotherapy (IMRT) were used in the patients. All patients were treated with a total dose of 50-70 Gy (1.8-2 Gy per fraction, 1 fraction per day, and 5 days per week). The radiation parameters were as follows: (A) energy, $6 \mathrm{MV}$ X-rays linear accelerator; (B) The gross tumor volume (GTV), including primary tumor and involved lymph nodes; (C) The clinical target volume (CTV) comprised GTV with at least $3 \mathrm{~cm}$ superior and inferior margins and at least $0.5 \mathrm{~cm}$ lateral margins; (D) The planned target volume (PTV), including a minimum of $0.5-1 \mathrm{~cm}$ surrounding the CTV. The dose and volume constraints for normal tissues were as follows: to the spinal cord, $<45 \mathrm{~Gy}$; to the heart, $\mathrm{V} 40<40 \%$; And for the Bilung, average dose (MLD) $\leq 18 \mathrm{~Gy}, \mathrm{~V} 5 \leq 65 \%, \mathrm{~V} 20 \leq 30 \%$.

\section{Chemotherapy}

All of the 581 eligible patients had received $0-8$ courses of concurrent or sequential chemotherapy. We used antiemetic drugs to prevent vomiting in the course of chemotherapy. The chemotherapy regimens were based on platinum, including (A) docetaxel $75 \mathrm{mg} / \mathrm{m} 2 \mathrm{~d} 1$ or paclitaxel $135 \mathrm{mg} / \mathrm{m} 2 \mathrm{~d} 1+$ nedaplatin $75 \mathrm{mg} / \mathrm{m} 2 \mathrm{~d} 2$ or cisplatin $75 \mathrm{mg} / \mathrm{m} 2 \mathrm{~d} 2$ or lobaplatin $50 \mathrm{mg} \mathrm{d} 2$ or carboplatin AuC2 d2; (B) 5-fluorouracil (5-FU) 700-1000 mg/ $\mathrm{m} 2 \mathrm{~d} 1-2+$ cisplatin $75 \mathrm{mg} / \mathrm{m} 2 \mathrm{~d} 2$. Before and after every 
cycle of chemotherapy, complete blood biochemical data was obtained. Once severe toxicity happened, the chemotherapy dose would be adjusted in the next cycle.

\section{Toxicity}

Toxicity was graded according to the Radiation Therapy Oncology Group (RTOG)/European Organization for Research and Treatment of Cancer radiation morbidity score system (EORTC). The grade of toxicity was scored retrospectively based on the medical records. An adverse effect at more than 3 months after completion of radiotherapy was defined as late toxicity. The toxicity was recorded as a maximum grade at any time during the treatment or follow-up period. Once severe radiation esophagitis/ pneumonitis/ hematologic toxicity appeared, both chemotherapy and radiotherapy would stop until recovery.

Definition of the nutritional index and inflammatory index The serum albumin level $(\mathrm{g} / \mathrm{L})+5$ multiplied by the absolute lymphocytes count to calculate the prognostic nutrition index (PNI). The absolute neutrophils count divided by the absolute lymphocytes count to calculate the neutrophils-lymphocytes ratio (NLR). The absolute platelets count divided by the absolute lymphocytes count was used to calculate the platelets-lymphocytes ratio (PLR). The absolute lymphocytes count divided by the absolute monocytes count to calculate the lymphocytes-monocytes ratio (LMR). Finally, the absolute platelets count multiplied by NLR to calculate the systemic immuneinflammation index (SII) [15].

\section{Evaluation methods and follow-up}

All patients were followed up to detect survival status and disease progression every 3 months during the first years, every 6 months in the following 2 years, and once a year later until the end of the study. Follow-up involved physical examination, blood tests, biochemistry, tumor markers, upper gastrointestinal endoscopy, barium esophagography, CT, or PET-CT. Information about survival status and disease progression was updated until April 2021. The endpoint of the study was overall survival (OS) and progression-free survival (PFS). The OS is defined as the period from pathological diagnosis to death or the last follow-up. The PFS is defined as the period from pathological diagnosis to tumor progression, death, or last follow-up. The follow-up information came from the patient's clinical charts and / or telephone interviews. The median duration of follow-up was 23.2 months (range, 2.0 to 162.6 months).

\section{Statistical analysis}

All statistical analyses were performed using $\mathrm{R}$ software (version 4.0.2) and SPSS software (version 26.0). The optimal cutoff values of radiotherapy (RT) dose, tumor length, tumor thickness, PNI, NLR, PLR, LMR, and SII are calculated using the X-tile application (https://medicine.yale. $\mathrm{edu} / \mathrm{lab} / \mathrm{rimm} / \mathrm{research} / \mathrm{software} /)$. Categorical data were compared by the Chi-square test or the Fisher exact test. Continuous variables were compared using the MannWhitney $U$ test. The survival curve was drawn using the Kaplan-Meier method. The Cox regression model was used for univariate and multivariate analysis. In univariate analysis, all factors with $p<0.05$ were involved in multivariate analysis to determine independent prognostic factors. All analyses were two-sided, and a $p$ value $<0.05$ was considered to be statistically significant.

\section{Results}

\section{Patient stratification}

The baseline characteristics of our study patients are shown in Table 1. A total of 581 patients underwent definitive chemoradiotherapy (dCRT), of whom 317 (54.6\%) received chemotherapy and 264 (45.4\%) did not. Only 101 (17.4\%) patients received RT dose $\leq 58.8$ Gy, and 480 patients received $>58.8$ Gy. There were 316 (54.6\%) patients aged 65-74 years and 265 (45.4\%) aged 75 years or older. The median age of the patients was 74 years old (range, 65-90 years old). Overall, 373 (64.2\%) were male, 148 (25.5\%) were stage II, 180 (31\%) were stage III, and 253 (43.5\%) were stage IVA. Those treated with chemotherapy tended to be younger, were more likely to have stage IVA. The optimal cutoff value for RT dose, tumor length, tumor thickness, PNI, NLR, PLR, LMR, and SII was calculated to be 58.8, 5.8, 1.2, $41.7,4.44,180,3.73$, and 918 , respectively.

\section{Overall survival in different age groups}

The median overall survival (OS) of the overall population was 23.2 months (range, $2.0-162.6$ months), and 1 , 3 , and 5 years OS rates were $79.3 \%, 43.7 \%$, and $31.7 \%$, respectively. For patients treated with chemoradiotherapy (CRT) and radiotherapy (RT) alone, the median OS was 25 months (range, 2.9-124.4 months) and 19.5 months (range, 2.0-162.6 months), respectively $(p<0.01)$. The 1,3 , and 5 years OS rates in patients treated with CRT were $83.3 \%, 48.5 \%$, and $36.7 \%$, respectively, while in patients treated with RT alone were $74.5 \%, 38.0 \%$, and $25.7 \%$, respectively.

\section{Progression-free survival in different age groups}

The median progression-free survival (PFS) of the overall population was 18.6 months (range, 
Table 1 Patients' characteristics of 581 locally advanced ESCC patients and patients' clinicopathological characteristics according to chemotherapy

\begin{tabular}{|c|c|c|c|c|c|}
\hline Clinicopathologic variable & & Total(N) & CRT $(n=317)$ & RT $(n=264)$ & $p$ value \\
\hline \multirow[t]{3}{*}{ Age (years) } & & & & & $<0.001$ \\
\hline & $65-74$ & $316(54.4 \%)$ & $245(42.2 \%)$ & $71(12.2 \%)$ & \\
\hline & $\geq 75$ & $265(45.6 \%)$ & $72(12.4 \%)$ & $193(33.2 \%)$ & \\
\hline \multirow[t]{3}{*}{ Gender } & & & & & 0.005 \\
\hline & Male & $373(64.2 \%)$ & $220(37.9 \%)$ & $153(26.3 \%)$ & \\
\hline & Female & $208(35.8 \%)$ & 97 (16.7\%) & 111 (19.1\%) & \\
\hline \multirow[t]{3}{*}{ Weight loss } & & & & & 0.023 \\
\hline & Yes & $281(48.4 \%)$ & 167 (28.7\%) & $114(19.6 \%)$ & \\
\hline & No & $300(51.6 \%)$ & $150(25.8 \%)$ & $150(25.8 \%)$ & \\
\hline \multirow[t]{3}{*}{ RT dose (Gy) } & & & & & 0.567 \\
\hline & $\leq 58.8$ & $101(17.4 \%)$ & $52(9.0 \%)$ & 49 (8.4\%) & \\
\hline & $>58.8$ & $480(82.6 \%)$ & $265(45.6 \%)$ & $215(37.0 \%)$ & \\
\hline \multirow[t]{5}{*}{ Tumor location } & & & & & 0.118 \\
\hline & Cervical & $37(6.4 \%)$ & $26(4.5 \%)$ & $11(1.9 \%)$ & \\
\hline & Upper thoracic & $133(22.9 \%)$ & $78(13.4 \%)$ & 55 (9.5\%) & \\
\hline & Middle thoracic & $338(58.2 \%)$ & $176(30.3 \%)$ & $162(27.9 \%)$ & \\
\hline & Lower thoracic & 73 (12.6\%) & $37(6.4 \%)$ & $36(6.2 \%)$ & \\
\hline \multirow[t]{3}{*}{ Tumor length (cm) } & & & & & 0.010 \\
\hline & $\leq 5.8$ & $344(59.2 \%)$ & $172(29.6 \%)$ & $172(29.6 \%)$ & \\
\hline & $>5.8$ & $237(40.8 \%)$ & 145 (25.0\%) & $92(15.8 \%)$ & \\
\hline \multirow[t]{3}{*}{ Tumor thickness (cm) } & & & & & 0.989 \\
\hline & $\leq 1.2$ & $232(39.9 \%)$ & $126(21.7 \%)$ & $106(18.2 \%)$ & \\
\hline & $>1.2$ & $349(60.1 \%)$ & 191 (32.9\%) & 158 (27.2\%) & \\
\hline \multirow[t]{4}{*}{ T stage } & & & & & 0.005 \\
\hline & $\mathrm{T} 2$ & 41 (7.1\%) & $16(2.8 \%)$ & 25 (4.3\%) & \\
\hline & T3 & 299 (51.5\%) & 152 (26.2\%) & 147 (25.3\%) & \\
\hline & $\mathrm{T} 4$ & 241 (41.5\%) & 149 (25.6\%) & 92 (15.8\%) & \\
\hline \multirow[t]{5}{*}{ N stage } & & & & & 0.030 \\
\hline & No & 190 (32.7\%) & 91 (15.7\%) & 99 (17.0\%) & \\
\hline & N1 & $259(44.6 \%)$ & 143 (24.6\%) & 116 (20.0\%) & \\
\hline & $\mathrm{N} 2$ & 111 (19.1\%) & 67 (11.5\%) & 44 (7.6\%) & \\
\hline & N3 & 21 (3.6\%) & $16(2.8 \%)$ & 5 (0.9\%) & \\
\hline \multirow[t]{4}{*}{ TNM stage } & & & & & 0.001 \\
\hline & Stage II & 148 (25.5\%) & $63(10.8 \%)$ & $85(14.6 \%)$ & \\
\hline & Stage III & $180(31.0 \%)$ & 96 (16.5\%) & $84(14.5 \%)$ & \\
\hline & Stage IVA & $253(43.5 \%)$ & 158 (27.2\%) & 95 (16.4\%) & \\
\hline \multirow[t]{3}{*}{ Year of diagnosis } & & & & & 0.059 \\
\hline & $2007-2017$ & 404 (69.5\%) & 210 (36.1\%) & 194 (33.4\%) & \\
\hline & $2018-2020$ & 177 (30.5\%) & 107 (18.4\%) & 70 (12.0\%) & \\
\hline \multirow[t]{3}{*}{ PNI } & & & & & 0.048 \\
\hline & $\leq 41.7$ & 88 (15.1\%) & 39 (6.7\%) & 49 (8.4\%) & \\
\hline & $>41.7$ & 493 (84.9\%) & $278(47.8 \%)$ & 215 (37.0\%) & \\
\hline \multirow[t]{3}{*}{ NLR } & & & & & 0.025 \\
\hline & $\leq 4.44$ & 517 (89.0\%) & 291 (50.1\%) & 226 (38.9\%) & \\
\hline & $>4.44$ & 64 (11.0\%) & $26(4.5 \%)$ & $38(6.5 \%)$ & \\
\hline \multirow[t]{3}{*}{ PLR } & & & & & 0.278 \\
\hline & $\leq 180$ & 451 (77.6\%) & 252 (43.4\%) & 199 (34.3\%) & \\
\hline & $>180$ & $130(22.4 \%)$ & 65 (11.2\%) & 65 (11.2\%) & \\
\hline
\end{tabular}


Table 1 (continued)

\begin{tabular}{|c|c|c|c|c|c|}
\hline Clinicopathologic variable & & Total(N) & CRT $(n=317)$ & $\mathrm{RT}(\mathrm{n}=264)$ & $p$ value \\
\hline \multirow[t]{3}{*}{ LMR } & & & & & 0.426 \\
\hline & $\leq 3.73$ & $246(42.3 \%)$ & $129(22.2 \%)$ & $117(20.1 \%)$ & \\
\hline & $>3.73$ & $335(57.7 \%)$ & $188(32.4 \%)$ & $147(25.3 \%)$ & \\
\hline \multirow[t]{3}{*}{ SII } & & & & & 0.149 \\
\hline & $\leq 918$ & $482(83.0 \%)$ & $270(46.5 \%)$ & $212(36.5 \%)$ & \\
\hline & $>918$ & $99(17.0 \%)$ & $47(8.1 \%)$ & $52(9.0 \%)$ & \\
\hline
\end{tabular}

ESCC, esophageal squamous cell carcinoma; CRT, chemoradiotherapy; RT, radiotherapy; T, tumor; N, node; TNM, tumor-node-metastasis; PNI, prognostic-nutrition index; NLR, neutrophils-lymphocytes ratio; PLR, platelets-lymphocytes ratio; LMR, lymphocytes-monocytes ratio; SII, systemic immune-inflammation index

1.1-159.6 months), and 1,3 , and 5 years PFS rates were $67.7 \%, 37.8 \%$, and $28.9 \%$, respectively. For patients treated with chemoradiotherapy (CRT) and radiotherapy (RT) alone, the median PFS was 20.4 months (range, 1.5-124.4 months) and 15.8 months (range, $1.1-159.6$ months), respectively $(\mathrm{p}<0.01)$. The 1,3 , and 5 years PFS rates in patients treated with CRT were $73.8 \%, 42.9 \%$, and $33.8 \%$, respectively, while in patients treated with RT alone were $60.4 \%, 31.8 \%$, and $23.2 \%$, respectively.

\section{Univariate and multivariate survival analysis of OS and PFS in ESCC}

Univariate and multivariate Cox regression analyses for predictors of OS and PFS was shown in Tables 2 and 3. In Table 2, univariate analyses revealed that the age $(p=0.009)$, chemotherapy $(p<0.001)$, RT dose $(p=0.025)$, tumor length $(p=0.002)$, tumor thickness $(p<0.001)$, N stage $(p<0.001)$, TNM stage $(p=0.006)$, PNI $(p<0.001)$, NLR $(p=0.001)$, PLR $(p=0.001)$, LMR $(p=0.004)$, and SII $(p<0.001)$ were significant risk factors for a worse OS. On multivariate analysis, the chemotherapy ( $p=0.007$; HR, $1.405 ; 95 \% \mathrm{CI}, 1.095-$ $1.804)$, tumor thickness ( $p=0.003$; HR, 1.429; 95\% CI, $1.126-1.813)$, and $\mathrm{N}$ stage $(p=0.011 ; \mathrm{HR}, 1.385 ; 95 \%$ CI, 1.076-1.784) were independently associated with worse OS. In Table 3, univariate analyses demonstrated that the age $(p=0.013)$, chemotherapy $(p=0.001)$, tumor length $(p=0.002)$, tumor thickness $(p<0.001), \mathrm{N}$ stage $(p<0.001)$, TNM stage $(p=0.002)$, Year of diagnosis $(p=0.028)$, PNI $(p<0.001)$, NLR $(p=0.001)$, PLR $(p=0.001), \operatorname{LMR}(p=0.001)$, and SII $(p<0.001)$ were significant risk factors for a worse PFS. On multivariate analysis, the chemotherapy $(p=0.022$; HR, 1.328; 95\% CI, 1.041-1.694), tumor thickness $(p=0.001 ; \mathrm{HR}$, $1.498 ; 95 \% \mathrm{CI}, 1.184-1.894)$, and $\mathrm{N}$ stage ( $p=0.006$; HR, 1.418; 95\% CI, 1.107-1.817) were independently associated with worse PFS. As shown in Fig. 1, there was a significant difference of OS and PFS in chemotherapy, tumor thickness, and $\mathrm{N}$ stage.

\section{Survival stratified by the cycle of chemotherapy}

We found that chemotherapy was an independent prognostic factor. In our study, 317 patients received 0-8 cycles of chemotherapy. In order to further analyze the relationship between the cycle of chemotherapy and OS and PFS, we classified the chemotherapy cycle into two categories and three categories. As shown in Fig. 2A-B, patients who received 2-8 cycles of chemotherapy had better OS than those who received 1 cycle of chemotherapy $(p=0.015)$, but PFS was not statistically significant $(p=0.126)$. However, Fig. $2 \mathrm{C}-\mathrm{D}$ showed that there were no significant differences in OS and PFS among the patients who received $1,2-3$, and $4-8$ cycles of chemotherapy $(p>0.05$ for all).

\section{Survival stratified by age}

We further analyzed the effects of age and dCRT on OS and PFS. Figure 3A-B showed that CRT has the better OS and PFS than RT alone for patients aged 65-74 years $(p=0.001$ and $p=0.002$, respectively). However, for patients aged 75 years or older, there was no statistically significant difference between CRT and RT alone in Fig. $3 C-\mathrm{D}(p=0.612$ and $p=0.652$, respectively).

\section{Survival stratified by age, applied therapy, and tumor stages}

We also analyzed the effects of age, applied therapy, and tumor stages on OS and PFS. Figure 4A-B showed that higher staged ESCC has the inferior OS and PFS than lower staged ESCC for patients received RT alone and aged $65-74$ years $(p=0.020$ for all and $p=0.006$ for all, respectively). However, for patients received CRT, there was no statistically significant difference between higher staged ESCC and lower staged ESCC in Fig. $4 C-\mathrm{D}(p=0.083$ for all and $p=0.167$ for all, respectively). Similarly, for patients received RT alone and aged 75 years or older, lower staged ESCC has the better PFS than higher staged ESCC, but not involve OS (Fig. 4E-F) $(p=0.063$ for all and $p=0.025$ for all, respectively). Finally, there was no statistically significant difference between lower staged ESCC and higher 
Table 2 Factors associated with overall survival: univariate and multivariate Cox proportional hazards models

\begin{tabular}{|c|c|c|c|c|c|c|}
\hline \multirow[t]{2}{*}{ Clinicopathologic parameters } & \multicolumn{3}{|c|}{ Univariate analysis } & \multicolumn{3}{|c|}{ Multivariate analysis } \\
\hline & $\mathrm{HR}$ & $95 \% \mathrm{Cl}$ & $p$ & $\mathrm{HR}$ & $95 \% \mathrm{Cl}$ & $p$ \\
\hline \multicolumn{7}{|l|}{ Age (years) } \\
\hline$\geq 75$ vs. $64-75$ & 1.314 & $1.069-1.614$ & 0.009 & 1.156 & $0.900-1.484$ & 0.256 \\
\hline \multicolumn{7}{|l|}{ Gender } \\
\hline Male vs. Female & 1.067 & $0.858-1.326$ & 0.560 & & & \\
\hline \multicolumn{7}{|l|}{ Weight loss } \\
\hline Yes vs. No & 0.918 & $0.747-1.129$ & 0.419 & & & \\
\hline \multicolumn{7}{|l|}{ Chemotherapy } \\
\hline No vs. Yes & 1.473 & $1.199-1.811$ & $<0.001$ & 1.405 & $1.095-1.804$ & 0.007 \\
\hline \multicolumn{7}{|l|}{ RT dose (Gy) } \\
\hline$\leq 58.8$ vs. $>58.8$ & 1.356 & $1.039-1.769$ & 0.025 & 1.185 & $0.902-1.558$ & 0.223 \\
\hline \multicolumn{7}{|l|}{ Tumor location } \\
\hline Cervical/Upper vs. Middle/Lower & 0.850 & $0.674-1.073$ & 0.171 & & & \\
\hline \multicolumn{7}{|l|}{ Tumor length $(\mathrm{cm})$} \\
\hline$>5.8$ vs. $\leq 5.8$ & 1.389 & $1.129-1.709$ & 0.002 & 1.226 & $0.984-1.528$ & 0.070 \\
\hline \multicolumn{7}{|l|}{ Tumor thickness (cm) } \\
\hline$>1.2$ vs. $\leq 1.2$ & 1.711 & $1.369-2.137$ & $<0.001$ & 1.429 & $1.126-1.813$ & 0.003 \\
\hline \multicolumn{7}{|l|}{ T stage } \\
\hline T4 vs. T2/T3 & 1.039 & $0.844-1.279$ & 0.716 & & & \\
\hline \multicolumn{7}{|l|}{$\mathrm{N}$ stage } \\
\hline N2/N3 vs. N0/N1 & 1.594 & $1.261-2.015$ & $<0.001$ & 1.385 & $1.076-1.784$ & 0.011 \\
\hline \multicolumn{7}{|l|}{ TNM stage } \\
\hline Stage III/Stage IV vs. Stage II & 1.415 & $1.102-1.817$ & 0.006 & 1.217 & $0.924-1.602$ & 0.162 \\
\hline \multicolumn{7}{|l|}{ Year of diagnosis } \\
\hline 2007-2017 vs. 2018-2020 & 1.153 & $0.873-1.522$ & 0.316 & & & \\
\hline \multicolumn{7}{|l|}{ PNI } \\
\hline$\leq 41.7$ vs. $>41.7$ & 1.669 & $1.273-2.189$ & $<0.001$ & 1.227 & $0.898-1.676$ & 0.199 \\
\hline \multicolumn{7}{|l|}{ NLR } \\
\hline$>4.44$ vs. $\leq 4.44$ & 1.672 & $1.226-2.281$ & 0.001 & 1.135 & $0.771-1.669$ & 0.521 \\
\hline \multicolumn{7}{|l|}{ PLR } \\
\hline$>180$ vs. $\leq 180$ & 1.517 & $1.197-1.924$ & 0.001 & 1.103 & $0.818-1.487$ & 0.520 \\
\hline \multicolumn{7}{|l|}{ LMR } \\
\hline$\leq 3.73$ vs. $>3.73$ & 1.357 & $1.103-1.668$ & 0.004 & 1.128 & $0.901-1.413$ & 0.293 \\
\hline \multicolumn{7}{|l|}{ SII } \\
\hline$>918$ vs. $\leq 918$ & 1.726 & $1.339-2.226$ & $<0.001$ & 1.267 & $0.897-1.790$ & 0.180 \\
\hline
\end{tabular}

$\mathrm{HR}$, hazard ratio; $\mathrm{Cl}$, confidence interval; RT, radiotherapy; T, tumor; N, node; TNM, tumor-node-metastasis; PNI, prognostic-nutrition index; NLR, neutrophilslymphocytes ratio; PLR, platelets-lymphocytes ratio; LMR, lymphocytes-monocytes ratio; SIl, systemic immune-inflammation index

staged ESCC of patients received CRT in Fig. 4G-H ( $p=0.284$ for all and $p=0.241$ for all, respectively).

\section{Toxicity}

Grade 3-4 radiation esophagitis (RE) was identified in $6.9 \%(22 / 317)$ of the CRT group and $10.2 \%(27 / 264)$ of the RT alone group in Table $4((p=0.158$; OR, $1.528 ; 95 \%$ CI, $0.848-2.751))$. Of the 581 patients, radiation pneumonitis (RP) occurred in $29(9.1 \%)$ and 19 (7.1\%) patients of CRT group and RT alone group $((p=0.396 ; \mathrm{OR}, 0.770$; 95\% CI, $0.421-1.408)$ ). Hematologic toxicity grade $\geq 3$ was observed in $51(16.1 \%)$ and $7(2.7 \%)$ of patients received CRT and $\mathrm{RT}$ alone, respectively $((p<0.001$; OR, 0.142; 95\% CI, 0.063-0.319)).

\section{Discussion}

There is still no definitely therapeutic evidence of a beneficial effect of chemotherapy with radiotherapy for older patients with locally advanced esophageal squamous cell carcinoma (ESCC). Since the Radiation Therapy Oncology Group trial 85-01 indicated that the outcome of CRT was significantly better than that of RT alone for patients 
Table 3 Factors associated with progression-free survival: univariate and multivariate Cox proportional hazards models

\begin{tabular}{|c|c|c|c|c|c|c|}
\hline \multirow[t]{2}{*}{ Clinicopathologic parameters } & \multicolumn{3}{|c|}{ Univariate Analysis } & \multicolumn{3}{|c|}{ Multivariate Analysis } \\
\hline & $\mathrm{HR}$ & $95 \% \mathrm{Cl}$ & $p$ & HR & $95 \% \mathrm{Cl}$ & $p$ \\
\hline \multicolumn{7}{|l|}{ Age (years) } \\
\hline$\geq 75$ vs. $64-75$ & 1.292 & $1.056-1.581$ & 0.013 & 1.217 & $0.955-1.550$ & 0.112 \\
\hline \multicolumn{7}{|l|}{ Gender } \\
\hline Male vs. Female & 1.158 & $0.935-1.435$ & 0.178 & & & \\
\hline \multicolumn{7}{|l|}{ Weight loss } \\
\hline Yes vs. No & 0.960 & $0.784-1.175$ & 0.692 & & & \\
\hline \multicolumn{7}{|l|}{ Chemotherapy } \\
\hline No vs. Yes & 1.416 & $1.157-1.732$ & 0.001 & 1.328 & $1.041-1.694$ & 0.022 \\
\hline \multicolumn{7}{|l|}{ RT dose (Gy) } \\
\hline$\leq 58.8$ vs. $>58.8$ & 1.200 & $1.921-1.564$ & 0.176 & & & \\
\hline \multicolumn{7}{|l|}{ Tumor location } \\
\hline Cervical/Upper vs. Middle/Lower & 0.867 & $0.691-1.088$ & 0.219 & & & \\
\hline \multicolumn{7}{|l|}{ Tumor length $(\mathrm{cm})$} \\
\hline$>5.8$ vs. $\leq 5.8$ & 1.377 & $1.124-1.687$ & 0.002 & 1.179 & $0.950-1.465$ & 0.135 \\
\hline \multicolumn{7}{|l|}{ Tumor thickness (cm) } \\
\hline$>1.2$ vs. $\leq 1.2$ & 1.830 & $1.472-2.276$ & $<0.001$ & 1.498 & $1.184-1.894$ & 0.001 \\
\hline \multicolumn{7}{|l|}{ T stage } \\
\hline T4 vs. T2/T3 & 1.109 & $0.906-1.359$ & 0.316 & & & \\
\hline \multicolumn{7}{|l|}{$\mathrm{N}$ stage } \\
\hline N2/N3 vs. N0/N1 & 1.589 & $1.262-2.001$ & $<0.001$ & 1.418 & $1.107-1.817$ & 0.006 \\
\hline \multicolumn{7}{|l|}{ TNM stage } \\
\hline Stage III/Stage IV vs. Stage II & 1.485 & $1.161-1.900$ & 0.002 & 1.286 & $0.981-1.687$ & 0.069 \\
\hline \multicolumn{7}{|l|}{ Year of diagnosis } \\
\hline 2007-2017 vs. 2018-2020 & 1.345 & $1.033-1.752$ & 0.028 & 1.293 & $0.986-1.695$ & 0.063 \\
\hline \multicolumn{7}{|l|}{ PNI } \\
\hline$\leq 41.7$ vs. $>41.7$ & 1.682 & $1.290-2.193$ & $<0.001$ & 1.237 & $0.912-1.677$ & 0.172 \\
\hline \multicolumn{7}{|l|}{ NLR } \\
\hline$>4.44$ vs. $\leq 4.44$ & 1.659 & $1.221-2.255$ & 0.001 & 1.216 & $0.839-1.762$ & 0.301 \\
\hline \multicolumn{7}{|l|}{ PLR } \\
\hline$>180$ vs. $\leq 180$ & 1.507 & $1.193-1.902$ & 0.001 & 1.140 & $0.849-1.529$ & 0.384 \\
\hline \multicolumn{7}{|l|}{ LMR } \\
\hline$\leq 3.73$ vs. $>3.73$ & 1.417 & $1.157-1.734$ & 0.001 & 1.161 & $0.932-1.447$ & 0.184 \\
\hline \multicolumn{7}{|l|}{ SII } \\
\hline$>918$ vs. $\leq 918$ & 1.715 & $1.335-2.203$ & $<0.001$ & 1.246 & $0.890-1.744$ & 0.200 \\
\hline
\end{tabular}

$\mathrm{HR}$, hazard ratio; $\mathrm{Cl}$, confidence interval; RT, radiotherapy; T, tumor; N, node; TNM, tumor-node-metastasis; PNI, prognostic-nutrition index; NLR, neutrophilslymphocytes ratio; PLR, platelets-lymphocytes ratio; LMR, lymphocytes-monocytes ratio; SIl, systemic immune-inflammation index

with esophageal cancer [10], CRT has been a standard treatment for esophageal carcinoma (EC). However, most of these studies typically included few patients aged 65 years or older. Besides, most elderly patients are unable to tolerate chemotherapy. Whether the therapeutic regimens from younger patients can be applied to elderly patients remains controversial, and data are scarcely available.

This study aimed to review our hospital's experience among the elderly patients with locally advanced ESCC who were treated with definitive chemoradiotherapy
(dCRT) to better understand the efficacy of this approach in comparison to radiotherapy (RT) alone. To the best of our knowledge, our study has a relatively large sample of elderly patients aged 65 years or older for comparison. In the study, compared to the radiotherapy (RT) group, the chemoradiotherapy (CRT) group had significantly better overall survival (OS) and progression-free survival (PFS). Furthermore, multivariate Cox analysis showed that chemotherapy, tumor thickness, and $\mathrm{N}$ stage were regarded as independent prognostic factors that affect both the OS and PFS. 
A
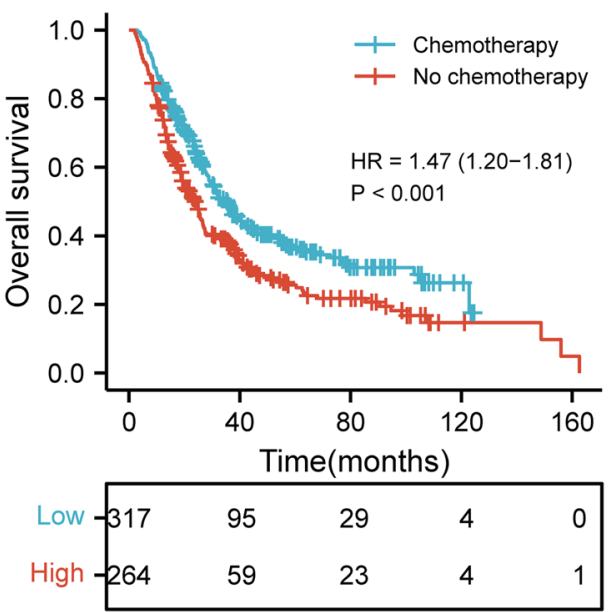

B
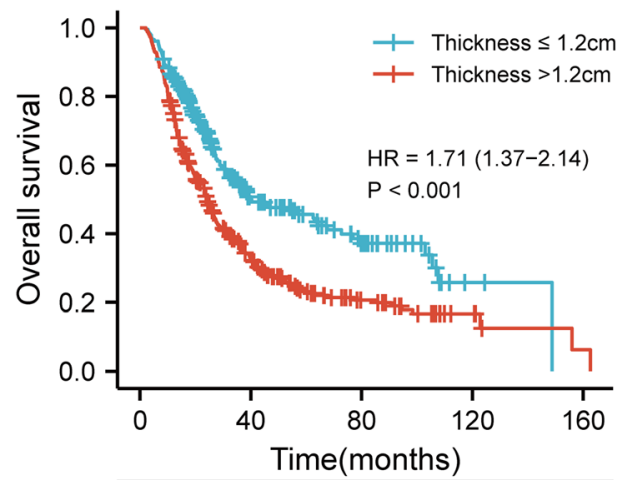

Low \begin{tabular}{rllll|}
-232 & 67 & 25 & 2 & 0 \\
-349 & 87 & 27 & 6 & 1 \\
\hline
\end{tabular}

C

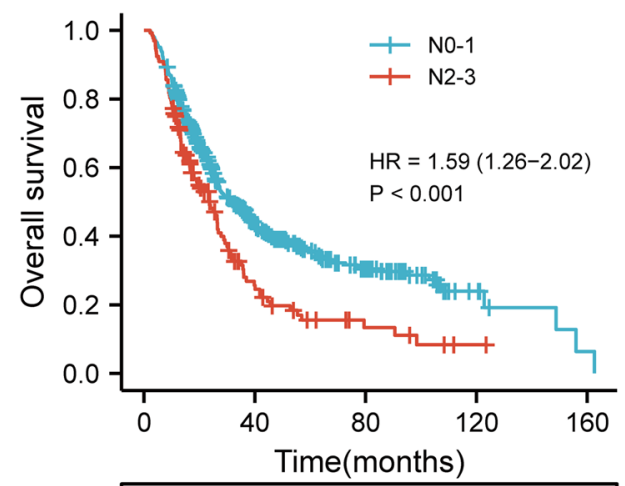

High -\begin{tabular}{ccccc|}
449 & 132 & 46 & 7 & 1 \\
132 & 22 & 6 & 1 & 0 \\
\hline
\end{tabular}

$\mathrm{D}$

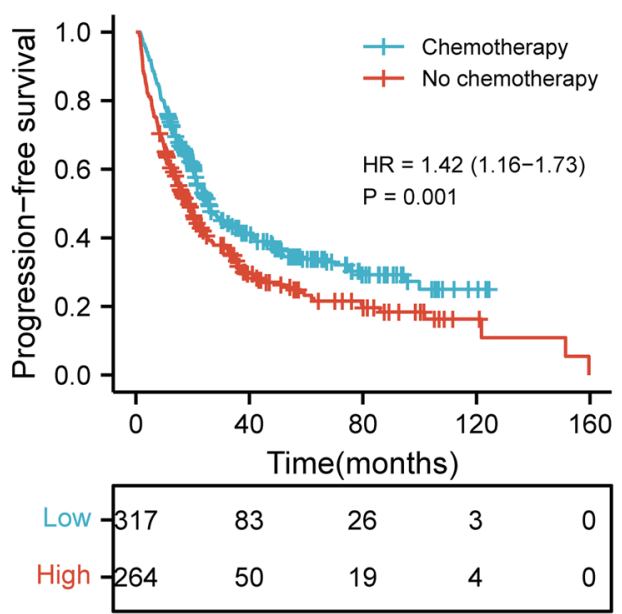

$\mathrm{E}$

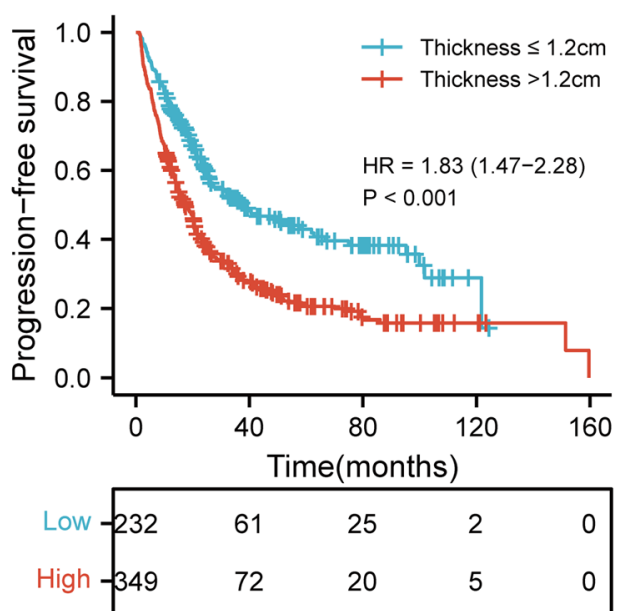

$\mathrm{F}$

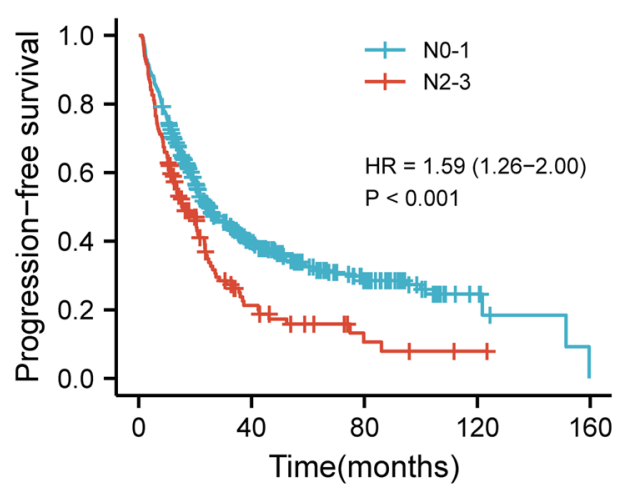

High -\begin{tabular}{ccccc|}
449 & 116 & 41 & 6 & 0 \\
132 & 17 & 4 & 1 & 0 \\
\hline
\end{tabular}

Fig. 1 Kaplan-Meier curves of chemotherapy, tumor thickness, and N stage for the whole study population showing (A-C) overall survival $(p<0.001, p<0.001, p<0.001$, respectively); (D-F) progression-free survival $(p=0.001, p<0.001, p<0.001$, respectively). HR, hazard ratio; $\mathrm{N}$, node 

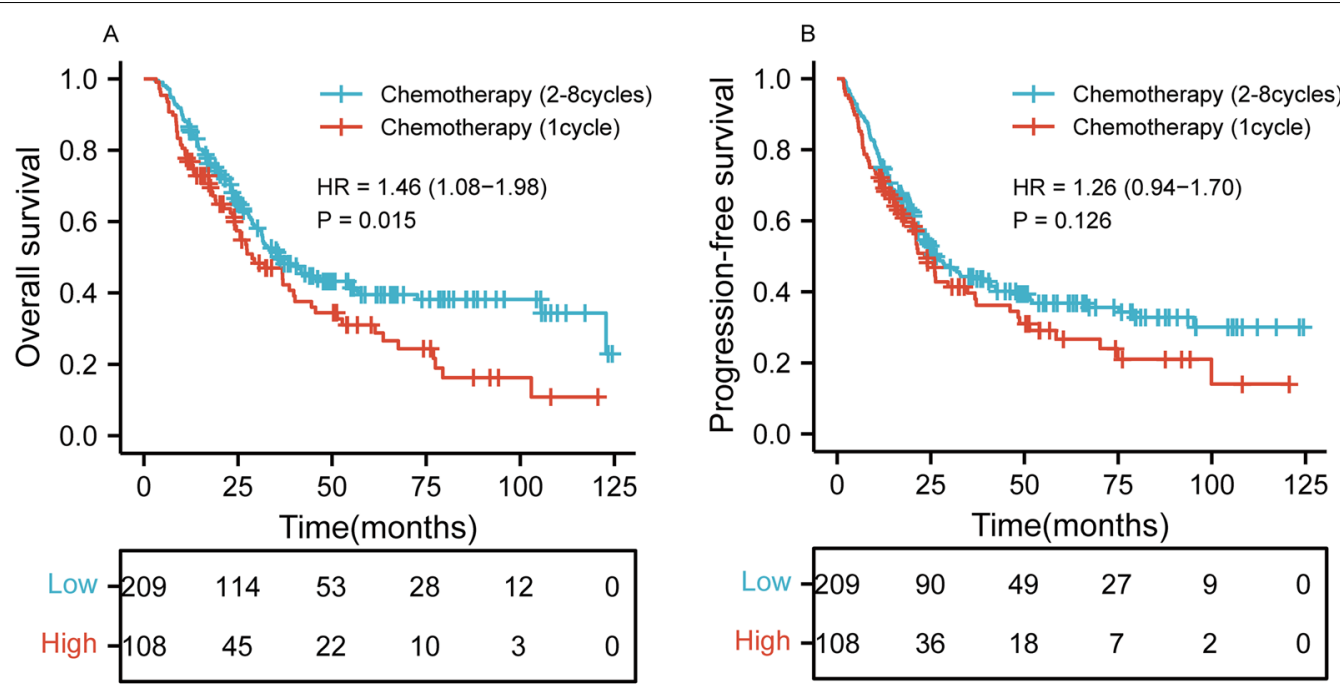

C

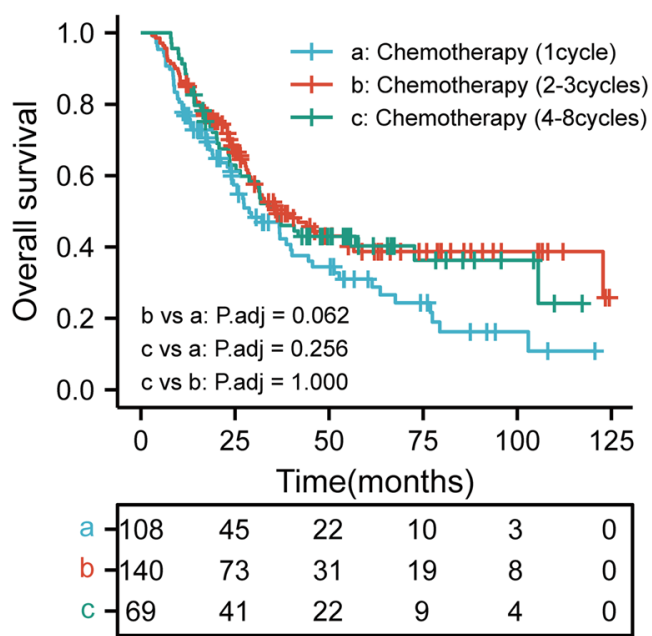

D

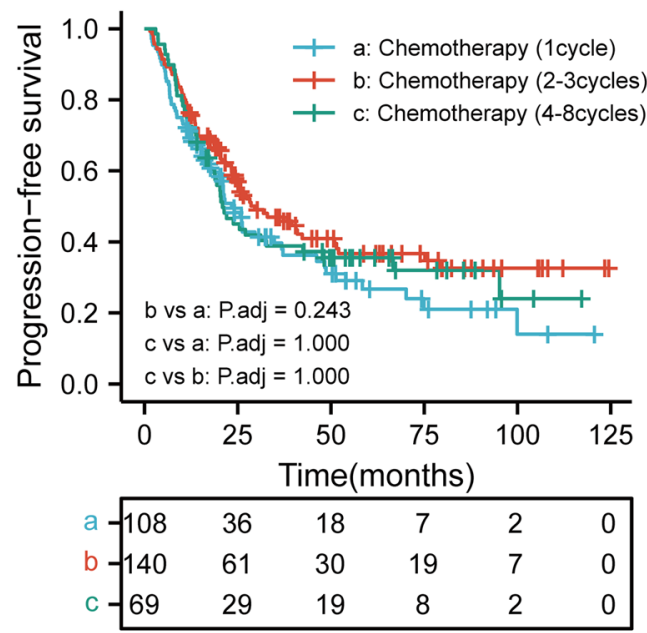

Fig. 2 Kaplan-Meier curves according to cycle of chemotherapy categories among patients who received chemotherapy showing A-B overall survival $(p=0.015)$ and progression-free survival $(p=0.126)$ for 1 and $2-8$ cycles of chemotherapy; C-D overall survival $(p>0.05)$ and progression-free survival $(p>0.05$ ) for 1, 2-3, and 4-8 cycles of chemotherapy. HR, hazard ratio; Adj, adjust

Several studies showed that CRT is an effective treatment in elderly patients with EC [16-19], which is similar to our multivariate analysis result. Whether CRT is effective and tolerable for elderly patients $(>65$ years or $\geq 70$ years) were analyzed by two retrospective studies which revealed significant results of CRT with a better survival benefit, which suggested that CRT can be effective and feasible for older patients [13, 17]. However, the sample size of these studies is relatively small. Additionally, most of these study populations are aged $75 / 80$ years or older.

$\mathrm{N}$ stage and tumor thickness were found to be independent predictors affecting both OS and PFS in our study. As is known to us all, an increased number of positive lymph nodes and an increase in the lymph node classification were associated with a worse prognosis in esophageal carcinoma [20]. Although some studies demonstrated that tumor thickness is an independent prognostic factor for EC, there is still no consensus on the prognostic cutoff point of tumor thickness21, 22. Our study showed that the optimum cutoff value of tumor thickness was $1.2 \mathrm{~cm}$, which is different from previous studies $[21,22]$. The sample size and measuring method are the two crucial reasons for the inconsistency of the results.

In order to further analyze the relationship between the cycle of chemotherapy and OS and PFS, we classified the chemotherapy cycle into two categories and three 

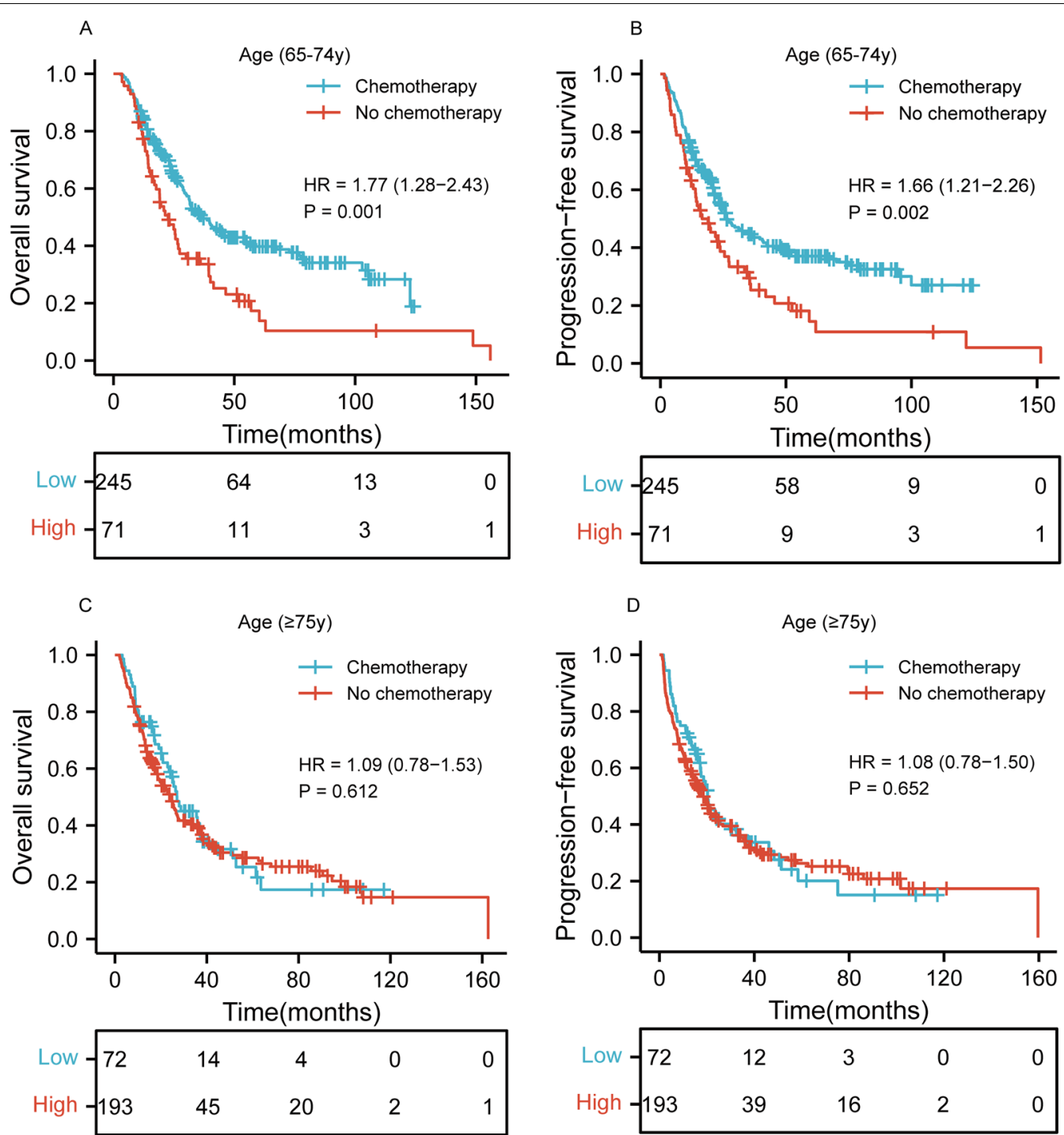

Fig. 3 Kaplan-Meier curves according to age categories for the whole study population showing $\mathbf{A}-\mathbf{B}$ overall survival $(p=0.001)$ and progression-free survival $(p=0.002)$ of patients aged $65-74$ years; $\mathbf{C}-\mathbf{D}$ overall survival $(p=0.612)$ and progression-free survival $(p=0.652)$ of patients aged 75 years or older. HR, hazard ratio

categories. The result showed that patients who received 2-8 cycles of chemotherapy had better OS than those who received 1 cycle of chemotherapy $(p=0.015)$, but PFS was not statistically significant $(p=0.126)$. In addition, there were no significant differences in OS and PFS among the patients who received $1,2-3$, and $4-8$ cycles of chemotherapy ( $p>0.05$ for all). It suggested that for patients receiving chemotherapy, 2 or more cycles of chemotherapy may be related to better OS.

\section{(See figure on next page.)}

Fig. 4 Kaplan-Meier curves according to age, applied therapy, and tumor stages categories for the whole study population showing A-B overall survival ( $p=0.020$ for all) and progression-free survival ( $p=0.006$ for all) of patients received RT alone and aged $65-74$ years in different tumor stages; C-D overall survival ( $p=0.083$ for all) and progression-free survival ( $p=0.167$ for all) of patients received CRT and aged $65-74$ years in different tumor stages; $\mathbf{E}-\mathbf{F}$ overall survival ( $p=0.063$ for all) and progression-free survival ( $p=0.025$ for all) of patients received RT alone and aged 75 years or older in different tumor stages; G-H overall survival ( $p=0.284$ for all) and progression-free survival $(p=0.241$ for all) of patients received CRT and aged 75 years or older in different tumor stages. RT, radiotherapy; CRT, chemoradiotherapy 

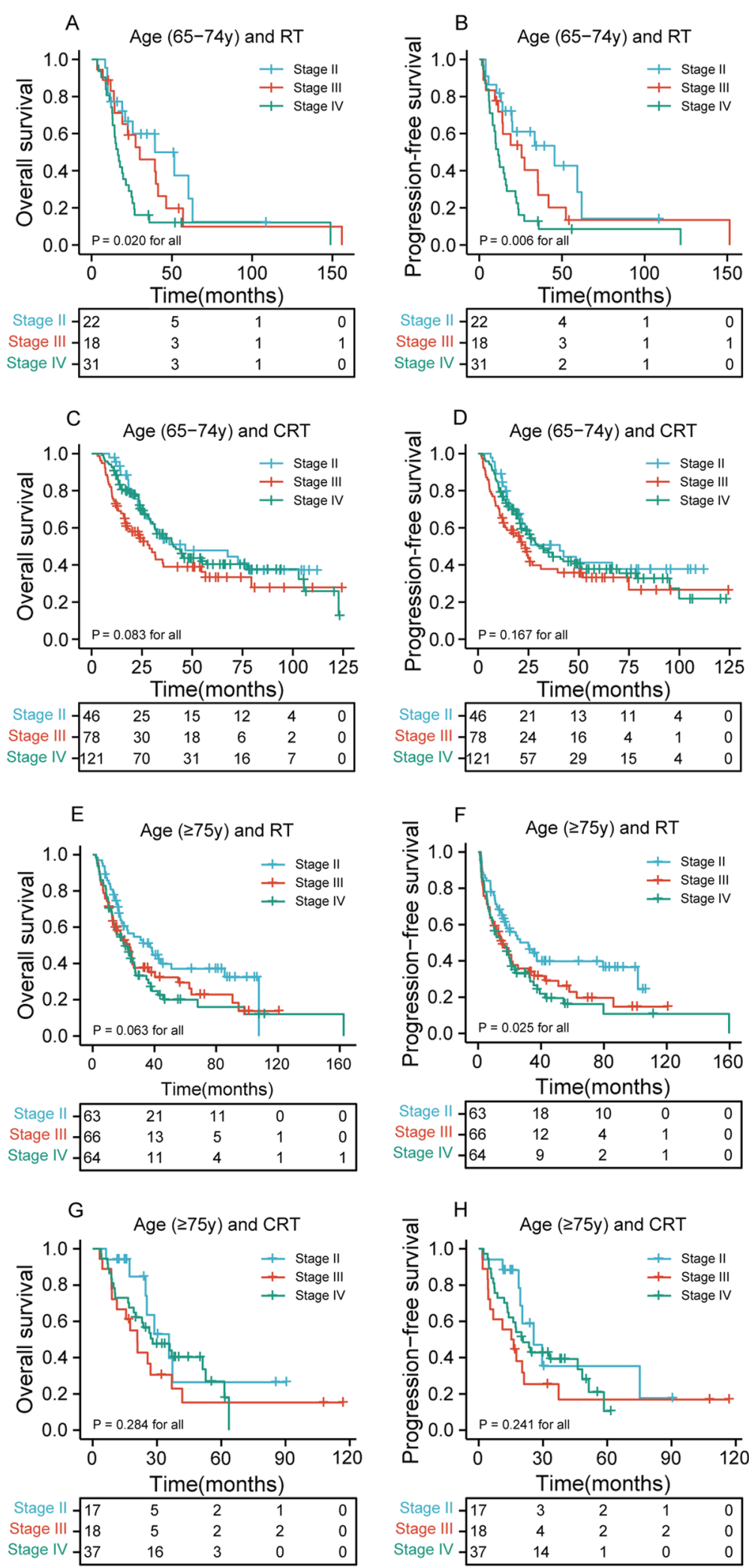

Fig. 4 (See legend on previous page.) 
Table 4 Toxicities of chemoradiotherapy and radiotherapy alone for all patients

\begin{tabular}{lccr}
\hline Toxicity & $\begin{array}{l}\text { CRT group } \\
(\mathbf{n}=\mathbf{3 1 7})\end{array}$ & $\begin{array}{l}\text { RT group } \\
(\mathbf{n}=\mathbf{2 6 4})\end{array}$ & $\boldsymbol{p}$ value \\
\hline Radiation esophagitis & & & 0.158 \\
Grade $<3$ & 295 & 237 & \\
Grade $\geq 3$ & 22 & 27 & 0.396 \\
Radiation pneumonitis & & & \\
No & 288 & 245 & $<0.001$ \\
Yes & 29 & 19 & \\
Hematologic toxicity & & & \\
Grade $<3$ & 266 & 257 & \\
Grade $\geq 3$ & 51 & 7 & \\
\hline CRT & & &
\end{tabular}

$\mathrm{CRT}$, chemoradiotherapy; $\mathrm{RT}$, radiotherapy

Many studies have shown that age was not a risk factor of survival, which is similar to our result $[13,17,23]$. A previous study showed that life expectancy is over 10 years at 70 years of age [24]. Therefore, older patients with ESCC may benefit from curative treatment. Nonetheless, many physicians are hesitant to deliver curative CRT to elderly patients because of its severe toxicity in reality. In general, older patients are unlikely to tolerate chemotherapy [25, 26]. However, some studies suggested that CRT in older patients had survival benefits compared with RT alone [19, 27-29]. Conversely, Jingu et al. and Zhou et al. indicated that CRT for esophageal cancer in patients ( $\geq 80$ years or $\geq 75$ years) did not have statistically significant survival benefit from CRT compared with RT alone [30,31]. In the present study, we further analyzed the effects of age and dCRT on OS and PFS. The results indicated that CRT has the better OS and PFS than RT alone for patients aged 65-74 years (both $p<0.01$ ). Interestingly enough, for patients aged 75 years or older, there was no statistically significant difference between the CRT group and RT alone group (both $p>0.05$ ). It suggested that CRT may have no benefit for elderly ESCC patients aged 75 years or older than RT alone.

As is known to all, higher staged cancers and milder types of therapy are identified as factors of poorer prognosis in EC. To clearly elaborate influence of the therapeutic effect on survival and course of disease, we also analyzed the effects of age, applied therapy, and tumor stages on OS and PFS. The result showed that higher staged ESCC has the inferior OS and PFS than lower staged ESCC for patients received RT alone and aged 65-74 years $(p<0.05)$. However, for patients received CRT, there was no statistically significant difference between higher staged ESCC and lower staged ESCC $(p>0.05)$. Similarly, for patients received RT alone and aged 75 years or older, lower staged ESCC has the better PFS than higher staged ESCC $(p<0.05)$.

So far, there is no evidence to suggest that advanced age is an independent contraindication for CRT in the retrospective studies. In our study, 317 (54.6\%) patients received CRT compared to the RT alone (45.4\%). The rate of grade $\geq 3$ radiation esophagitis (RE) in the CRT group and the RT group had no statistical significance $(p=0.158)$. There was also no statistical difference in the incidence of radiation pneumonitis (RP) $(p=0.396)$. However, there was significantly more severe hematologic toxicity in the CRT group than in that treated with RT alone in this study $(p<0.001)$. In addition, several studies indicated that older age was found to be a risk factor for toxicity after dCRT for EC [32-35]. CRT should be conducted with special care for elderly patients. Our result showed that RT alone is regarded to be relatively safe. However, even RT alone requires special attention in elderly patients. Therefore, the potential benefit from CRT or RT should be carefully balanced against the risk of toxicity in elderly patients. Further research is needed to establish predictors that can identify risk factors for older patients and develop different selections for candidates who would benefit from different effective treatments.

Due to the retrospective nature, our study suffers from limitations. Firstly, this was a single institutional study, which may be subject to selection bias. Secondly, our study is limited to patients with ESCC and has no guiding significance for patients with other types of EC. Thirdly, RT dose and cycles of chemotherapy were different according to patients. Finally, as the study is, as clearly stated by us, of retrospective character, data is not powered towards selection of certain chemotherapy protocols in every aspect due to multiple protocols applied within study period. Thus, neglecting effects of chemotherapy in an older aged cohort may not possible though data shows no significance in overall survival/progression free survival in patients aged $>75$ years. This topic has clearly to be addressed in prospective trails with regard to different protocols and treatment toxicity. Although to our best knowledge, this is the relatively large series reported for this population in ESCC, the sample size is still quite small. Well-designed prospective studies in larger sample size are needed to corroborate our results.

\section{Conclusions}

In conclusion, the present study suggested that CRT for ESCC in patients aged 65 years or older had a significant benefit over RT alone in terms of OS and PFS. However, for patients aged 75 years or older, there was no statistically significant difference between CRT group and $\mathrm{RT}$ alone group. CRT should be performed with special 
attention in patients aged 75 years or older. High-level prospective clinical trials are needed, thus to offer a scientific basis for clinical therapy for elderly patients with locally advanced ESCC.

\begin{abstract}
Abbreviations
ESCC: Esophageal squamous cell carcinoma; CRT: Chemoradiotherapy; RT: Radiotherapy; OS: Overall survival; PFS: Progression-free survival; EC: Esophageal carcinoma; dCRT: Definitive chemoradiotherapy; TNM: Tumor-nodemetastasis; CT: Computed tomography; PET-CT: Positron emission tomography-CT; IMRT: Intensity modulated radiotherapy; GTV: Gross tumor volume; CTV: Clinical target volume; PTV: Planned target volume; RTOG: Radiation Therapy Oncology Group; EORTC: European Organization for Research and Treatment of Cancer radiation morbidity score system; PNI: Prognostic nutrition index; NLR: Neutrophils-lymphocytes ratio; PLR: Platelets-lymphocytes ratio; LMR: Lymphocytes-monocytes ratio; SII: Systemic immune-inflammation index; HR: Hazard ratio; Cl: Confidence interval; OR: Odds ratio; RE: Radiation esophagitis; RP: Radiation pneumonitis.
\end{abstract}

\section{Acknowledgements}

We would like to thank all the investigators and patients and acknowledge the work of Jiancheng Li, which remarkably improved the quality of this paper.

\section{Authors' contributions}

$J \mathrm{~L}, \mathrm{HW}$, and $\mathrm{YY}$ designed this study. HW, $Y Y$ and $\mathrm{QZ}$ contributed to the data collection. TL, YW, and ZW analyzed the data. JL supervised the study. YY, $H Z$, $\mathrm{LL}$, and QZ wrote the manuscript. All authors read and approved the final manuscript.

\section{Funding}

This work was supported by the Joint Funds for the Innovation of Science and Technology, Fujian Province (Grant number: 2018Y9111) and the Financial Foundation of Fujian Province (Grant number: (2020)729).

\section{Availability of data and materials}

The data that support the findings of this study are available from the corresponding author upon reasonable request.

\section{Declarations}

\section{Ethical approval and consent to participate}

The current study was approved by the ethics committee of Fujian Medical University Cancer Hospital, Fuzhou, China (K2021-086-01) and conducted in accordance with the principles of the Declaration of Helsinki and its amendment. All patients provided written informed consent prior to treatment, and all the information was anonymized prior to analysis.

\section{Consent for publication}

The manuscript has been approved by all authors for publication.

\section{Competing interests}

The authors report that they have no conflicts of interest pertaining to this work.

Received: 25 April 2021 Accepted: 18 October 2021

Published online: 30 October 2021

\section{References}

1. Ferlay J, et al. Cancer incidence and mortality worldwide: sources, methods and major patterns in GLOBOCAN 2012. Int J Cancer. 2015;136(5):E359-86.

2. Siegel RL, Miller KD, Jemal A. Cancer statistics, 2020. CA Cancer J Clin. 2020;70(1):7-30.
3. Faiz $Z$, et al. Increased resection rates and survival among patients aged 75 years and older with esophageal cancer: a Dutch nationwide population-based study. World J Surg. 2012;36(12):2872-8.

4. Trivers KF, Sabatino SA, Stewart SL. Trends in esophageal cancer incidence by histology, United States, 1998-2003. Int J Cancer. 2008;123(6):1422-8.

5. Enzinger PC, Mayer RJ. Esophageal cancer. N Engl J Med. 2003;349(23):2241-52.

6. Chang AY, et al. Measuring population ageing: an analysis of the Global Burden of Disease Study 2017. Lancet Public Health. 2019;4(3):e159-67.

7. Partridge L, Deelen J, Slagboom PE. Facing up to the global challenges of ageing. Nature. 2018;561(7721):45-56.

8. Law S, et al. Predictive factors for postoperative pulmonary complications and mortality after esophagectomy for cancer. Ann Surg. 2004:240(5):791-800.

9. Lee KG, et al. Risk factors associated with complication following gastrectomy for gastric cancer: retrospective analysis of prospectively collected data based on the Clavien-Dindo system. J Gastrointest Surg. 2014;18(7):1269-77.

10. Cooper JS, et al. Chemoradiotherapy of locally advanced esophageal cancer: long-term follow-up of a prospective randomized trial (RTOG 85-01). Radiat Therapy Oncol Group JAMA. 1999;281(17):1623-7.

11. Smit JK, et al. Survival after definitive (chemo)radiotherapy in esophageal cancer patients: a population-based study in the north-East Netherlands. Ann Surg Oncol. 2013;20(6):1985-92.

12. Minsky BD, et al. INT 0123 (Radiation Therapy Oncology Group 94-05) phase III trial of combined-modality therapy for esophageal cancer: high-dose versus standard-dose radiation therapy. J Clin Oncol. 2002;20(5):1167-74.

13. Anderson $\mathrm{SE}$, et al. Combined modality chemoradiation in elderly oesophageal cancer patients. Br J Cancer. 2007;96(12):1823-7.

14. Hutchins $L F$, et al. Underrepresentation of patients 65 years of age or older in cancer-treatment trials. N Engl J Med. 1999;341(27):2061-7.

15. Dai Y, et al. Long-term impact of prognostic nutritional index in cervical esophageal squamous cell carcinoma patients undergoing definitive radiotherapy. Ann Transl Med. 2019;7(8):175.

16. $\mathrm{Xu} \mathrm{HY}$, et al. Safety and efficacy of radiation and chemoradiation in patients over 70 years old with inoperable esophageal squamous cell carcinoma. Oncol Lett. 2014;7(1):260-6.

17. Tougeron $D$, et al. Safety and outcome of definitive chemoradiotherapy in elderly patients with oesophageal cancer. Br J Cancer. 2008;99(10):1586-92.

18. Zhao Q, et al. Comparison of definitive chemoradiotherapy and radiotherapy alone in patients older than 75 years with locally advanced esophageal carcinoma: A retrospective cohort study. Medicine (Baltimore). 2017;96(35):e7920.

19. Li X, et al. Feasibility and efficacy of concurrent chemoradiotherapy in elderly patients with esophageal squamous cell carcinoma: a respective study of 116 cases from a single institution. Asian Pac J Cancer Prev. 2015:16(4):1463-9.

20. Rice TW, Patil DT, Blackstone EH. 8th edition AJCC/UICC staging of cancers of the esophagus and esophagogastric junction: application to clinical practice. Ann Cardiothorac Surg. 2017;6(2):119-30.

21. Xu H, et al. Prognostic value of tumor length and diameter for esophageal squamous cell cancer patients treated with definitive (chemo) radiotherapy: potential indicators for nonsurgical T staging. Cancer Med. 2019;8(14):6326-34

22. Chen C-Z. Long-term outcomes and prognostic factors for patients with esophageal cancer following radiotherapy. World I Gastroenterol. 2013;19(10):1639.

23. Nallapareddy $\mathrm{S}$, et al. Chemoradiation is a tolerable therapy for older adults with esophageal cancer. Anticancer Res. 2005;25(4):3055-60.

24. Arias E. United States life tables, 2009. Natl Vital Stat Rep. 2014;62(7):1-63.

25. Hurria $A$, et al. Predicting chemotherapy toxicity in older adults with cancer: a prospective multicenter study. J Clin Oncol. 2011;29(25):3457-65.

26. Hurria $A$, et al. Role of age and health in treatment recommendations for older adults with breast cancer: the perspective of oncologists and primary care providers. J Clin Oncol. 2008;26(33):5386-92.

27. Zhao $L$, et al. Radiotherapy alone or concurrent chemoradiation for esophageal squamous cell carcinoma in elderly patients. J Cancer. 2017;8(16):3242-50. 
28. Tougeron D, et al. Esophageal cancer in the elderly: an analysis of the factors associated with treatment decisions and outcomes. BMC Cancer. 2010;10:510.

29. Zhang $\mathrm{P}$, et al. Is there a benefit in receiving concurrent chemoradiotherapy for elderly patients with inoperable thoracic esophageal squamous cell carcinoma? PLoS ONE. 2014;9(8):e105270.

30. Jingu $\mathrm{K}$, et al. Is concurrent chemotherapy with radiotherapy for esophageal cancer beneficial in patients aged 80 years or older? Anticancer Res. 2019:39(8):4279-83

31. Zhou YC, et al. Aging-related prognosis analysis of definitive radiotherapy for very elderly esophageal cancer. Cancer Med. 2018;7(5):1837-44.

32. Ito $\mathrm{H}$, et al. Long-term complications of definitive chemoradiotherapy for esophageal cancer using the classical method. J Radiat Res. 2017:58(1):106-13.
33. Mak RH, et al. Toxicity and outcomes after chemoradiation for esophageal cancer in patients age 75 or older. Dis Esophagus. 2010;23(4):316-23.

34. Li QQ, et al. Definitive concomitant chemoradiotherapy with docetaxel and cisplatin in squamous esophageal carcinoma. Dis Esophagus. 2010;23(3):253-9.

35. Takeuchi $\mathrm{S}$, et al. A retrospective study of definitive chemoradiotherapy for elderly patients with esophageal cancer. Am J Clin Oncol. 2007;30(6):607-11.

\section{Publisher's Note}

Springer Nature remains neutral with regard to jurisdictional claims in published maps and institutional affiliations.
Ready to submit your research? Choose BMC and benefit from:

- fast, convenient online submission

- thorough peer review by experienced researchers in your field

- rapid publication on acceptance

- support for research data, including large and complex data types

- gold Open Access which fosters wider collaboration and increased citations

- maximum visibility for your research: over $100 \mathrm{M}$ website views per year

At BMC, research is always in progress.

Learn more biomedcentral.com/submissions 